\title{
Course of exfoliation and simplex glaucoma after primary trabeculectomy
}

\author{
Vladislav Popovic, Johan Sjöstrand
}

\begin{abstract}
Aim-To study the course of exfoliation and simplex glaucoma with respect to intraocular pressure (IOP) regulation and visual field survival after primary trabeculectomy.

Methods-Postoperative IOP regulation and complications were analysed prospectively in 95 patients. Mean follow up was 46 months. Visual field survival was studied by high pass resolution perimetry (HRP) in a subsample of 28 patients.

Results-Medical treatment was reinstated in $42 \%$ of exfoliation and in $36 \%$ of simplex glaucoma. In these patients, mean medicine free survival time, last untreated IOP, and mean IOP at the end of follow up were similar for both glaucoma types. Among patients with controlled postoperative IOP without added medication, mean IOP at the end of follow up was significantly lower in exfoliation glaucoma. Visual field deterioration and the pattern of complications were similar for both glaucoma types.

Conclusion-The effect of trabeculectomy on IOP regulation was good in both types of glaucoma, and somewhat better in exfoliation glaucoma. The magnitude of IOP lowering could not separate patients with continued visual field deterioration from those in whom visual fields remained stable. Visual field preservation was similar for both glaucoma types.

(Br F Ophthalmol 1999;83:305-310)
\end{abstract}

Trabeculectomy is the method of choice for surgical treatment of chronic open angle glaucoma. Long term follow up shows that this procedure alone can control intraocular pressure (IOP) for 10 years postoperatively in about $60 \%$ of cases. ${ }^{1}$ With medical treatment, the success rate for IOP control increases to over $90 \% .^{12}$

Exfoliation glaucoma causes about one half of all cases of chronic open angle glaucoma in Scandinavia. ${ }^{3}$ Its prognosis on medical treatment is worse in comparison with simplex glaucoma. ${ }^{4-7}$ Argon laser trabeculoplasty (ALT) has better short term efficacy in exfoliation glaucoma, but the long term results do not differ between the two glaucoma types. ${ }^{89}$ The poorer response to medical treatment in exfoliation glaucoma explains the larger proportion of this glaucoma type among patients undergoing surgical treatment.

Trabeculectomy has been reported to be effective in exfoliation glaucoma. ${ }^{10}$ However, there are only a few studies in which the results of trabeculectomy in exfoliation and simplex glaucoma are compared. Törnqvist and Drolsum $^{11}$ and Konstas et $a l^{12}$ found the response to trabeculectomy better in exfoliation than in simplex glaucoma. The follow up period in the study by Konstas et al ${ }^{12}$ was 6 months. In the study by Törnqvist and Drolsum, ${ }^{11}$ in which follow up time was $\leqslant 10$ years, no analysis was made of preoperative variables and their relation to the outcome of the operation.

The aim of the present study was to assess the regulation of IOP and the survival of visual field in exfoliation and simplex glaucoma after primary trabeculectomy. In addition, the following question was to be answered: is there any postoperative IOP that may separate patients at risk for continued visual field deterioration after operation from patients without such risk?

\section{Patients and methods}

Included in the study were 95 patients operated on with primary trabeculectomy between January 1990 and December 1995 at the Department of Ophthalmology, Sahlgrenska University Hospital, Mölndal, Sweden. Fifty one patients were inhabitants of Mölndal's community and 44 patients were from the neighbouring communities. These two groups of patients did not differ significantly with respect to mean age or mean IOP at operation, nor with respect to sex or glaucoma type. Mean follow up after operation was 46.2 (SD 17.9, range 14-84) months.

The criteria for inclusion were as follows: patients with exfoliation or simplex glaucoma, no surgery performed in the operated eye before trabeculectomy, and a minimum follow up of 14 months after operation. In cases where both eyes were operated only the first operated eye was included in the study. Patients with a history of trauma or uveitis in the operated eye were excluded from the study.

The major indications of trabeculectomy were as follows: unsatisfactorily controlled IOP $(\geqslant 22 \mathrm{~mm} \mathrm{Hg})$ despite maximum tolerable medical treatment $(75 \%)$, progression of visual field defects despite good control of IOP $(17 \%)$, side effects of medical treatment $(6 \%)$, progression of optic disc cupping (1\%), and unsatisfactory compliance with treatment $(1 \%)$. In some cases, there were multiple reasons for a more aggressive treatment.

All patients were operated with local anaesthesia on an outpatient basis by the first author. The operation technique was as follows: after insertion of the superior rectus bridle suture, a fornix based conjunctival flap 
was fashioned and a $0.3 \mathrm{~mm}$ thick limbus based trapezoid or rectangular scleral flap was raised. Paracentesis was done at the 9 o'clock position; a little volume of the aqueous escaped without visible shallowing of the anterior chamber. In this way the risk for hasty IOP decrease and flattening of the anterior chamber during the next phase is minimised. The anterior chamber was then penetrated under the scleral flap and an approximately $1 \times 3 \mathrm{~mm}$ block of limbal tissue was excised. Basal iridectomy was performed and the scleral flap was reposed in two ways. In the first 60 operated patients the scleral flap was secured with two to four isolated 10-0 nylon sutures, and in the last 35 patients with two isolated and one U-shaped releasable suture. In the first 48 operated patients the conjunctiva was sutured with one single 10-0 nylon suture at each end of the incision, while a continuous suture was used to secure the conjunctival incision in the last 47 patients. No antimetabolite therapy was used. Postoperatively, all patients were treated with topical dexamethasone (Isopto-Maxidex) three to four times daily for 5-6 weeks and atropine (Isopto-Atropine) twice daily for about 3 weeks. The first postoperative control for all patients was on the first postoperative day. If no serious complication was noted on this visit, the next two visits were planned 1 and 3 weeks after operation for patients operated on without releasable scleral flap sutures; for patients in whom these sutures were used, one extra control was planned on the fourth postoperative day.

All patients were followed up prospectively. Data were collected concerning patients' age, sex, glaucoma type, preoperative treatment as well as IOP, best corrected monocular visual acuity (VA) expressed as the minimum angle of resolution (MAR), occurrence of cataract, and results of visual field investigation before operation. Postoperatively, IOP, visual acuity, results of visual field investigation, and development of early and late complications were noted. Identification of the glaucoma type was made clinically by preoperative slit lamp investigation in mydriasis and by gonioscopy. Exfoliation glaucoma was diagnosed if exfoliation material was seen on the corneal endothelium, iris surface, angle structures of the anterior

Table 1 Patients'characteristics at operation according to glaucoma type

\begin{tabular}{llll}
\hline Characteristic & Exfoliation glaucoma & Simplex glaucoma & p Value \\
\hline Sex (n): & & & \\
$\quad$ Male & 21 & 24 & $0.102^{\star}$ \\
$\quad$ Female & 32 & 18 & \\
Age (years) (SD) & $73.7(8.3)$ & $69.2(9.1)$ & $0.014 \dagger$ \\
IOP & $28.8(6.1)$ & $24.4(5.0)$ & $0.000 \dagger$ \\
No of drops & $4.0(1.6)$ & $2.6(1.3)$ & $0.143+$ \\
No of medicines & $2.4(0.7)$ & 27 & $0.008 \dagger$ \\
ALT (n): & 36 & 15 & $0.828^{\star}$ \\
$\quad$ Yes & 17 & $6.8(4.5)$ & $0.019 \dagger$ \\
$\quad$ No & $4.9(3.5)$ & $0.3(0.5)$ & $0.657 \dagger$ \\
Duration to operation (years) & $0.4(0.4)$ & 25 & $0.159 \ddagger$ \\
LogMAR & & 17 & \\
Cataract (n): & 24 & 0 & \\
$\quad$ No cataract & 26 & & \\
$\quad$ Hazly stage & 3 & & \\
& & &
\end{tabular}

$\mathrm{p}$ Value $=$ significance level; IOP $=$ intraocular pressure; $\mathrm{ALT}=$ argon laser trabeculoplasty; MAR $=$ minimum angle of resolution; ${ }^{\star}$ Fisher's exact test, $\dagger t$ test, $\neq \chi^{2}$ test. chamber, pupillary margin, or lens capsule. No histopathological analysis of iris specimens was done.

The term primary trabeculectomy designates the first operation. Preoperative IOP refers to an average of the last three preoperative IOP measurements. IOP at last visit refers to an average of the last three postoperative measurements. An assessment of cataract grade from an early stage (the margin of the optic disc was clearly seen) to more advanced stages was made clinically (by slit lamp investigation and ophthalmoscopy with a dilated pupil) and all but the early stage were excluded from the visual field analysis.

Visual field was investigated by manual Goldmann perimetry or one of the two computerised perimetry techniques-namely, high pass resolution perimetry (HRP) (HighTech vision SCI AB, Göteborg, Sweden) and Competer (Bara Elektronik, Lund, Sweden). Of the computerised methods we preferred HRP perimetry and tried to use the same perimetry technique as long as possible during follow up of every patient. However, patients with extensive field defects and patients who did not cooperate in any of the computerised methods were tested with Goldmann perimetry. No selection of different methods on the basis of glaucoma type or for other reasons (for example, age) was made. Of all patients in the study, visual field was investigated with HRP technique in $45 \%$, Competer was used in $12 \%$, Goldmann perimetry in $32 \%$, and in $11 \%$ the perimetry technique at and after operation was not uniform.

Twenty eight of the patients in the study tested with HRP perimetry were chosen for analysis of visual field survival after operation. The inclusion criteria for visual field analysis were as follows: visual field investigation with HRP both at operation and during postoperative follow up; minimum field follow up 12 months; and minimum of two postoperative field investigations. Excluded from this study were patients with age related macular degeneration and patients with cataract denser than that of an early stage. However, cases which underwent cataract operation after trabeculectomy were again included in the follow up analysis. Mean follow up of visual fields after operation was 45.7 (SD 16.4) months (minimum 16, maximum 82 months). Investigation of visual field was performed on average 3.3 (range 2-6) times after operation.

Postoperative changes in global deviation (GD) and neural capacity (NC) were studied. GD is the mean deviation from the normal threshold values over all tested points, and is an indicator of severity of overall visual field deterioration. It is expressed in decibels (dB). NC is based upon the linear relation between the HRP thresholds expressed as MAR and retinal ganglion cell separations. ${ }^{13} \mathrm{NC}$ is expressed in percentage of average normal.

Data are expressed as mean (SD) (Tables $1-4)$. The $t$ test, Fisher's exact test, $\chi^{2}$ test, multiple variable correlation, and KaplanMeier analysis were used for statistical analysis. 


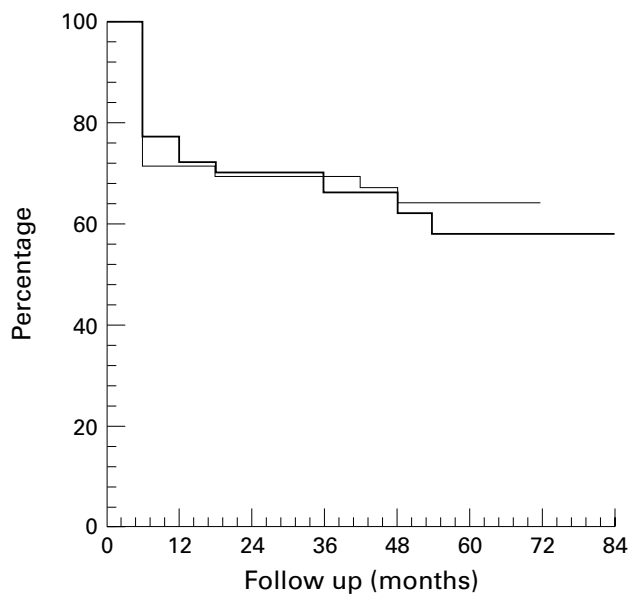

Figure 1 Medicine free time after trabeculectomy. Thick line $=$ exfoliation glaucoma, thin line $=$ simplex glaucoma .

\section{Results}

Preoperative characteristics of all patients in the study according to glaucoma type are given in Table 1. Kaplan-Meier survival analysis of medicine free time after operation for both glaucoma types is shown in Figure 1. Mean medicine free survival time in exfoliation glau-

Table 2 Characteristics of patients with added medication after operation (SD)

\begin{tabular}{lccc}
\hline Characteristic & Exfoliation glaucoma & Simplex glaucoma & p Value \\
\hline At operation: & & & \\
Sex (n): & 10 & 10 & $0.175^{\star}$ \\
$\quad$ Male & 12 & 5 & \\
Female & $72.0(7.8)$ & $69.3(7.7)$ & $0.309 \dagger$ \\
Age (years) & $29.0(7.3)$ & $29.3(4.7)$ & $0.877 \dagger$ \\
IOP & $4.2(1.6)$ & $4.3(1.5)$ & $0.697 \dagger$ \\
No of drops & $2.6(0.6)$ & $2.1(0.7)$ & $0.070 \dagger$ \\
No of medicines & & 10 & $0.600^{\star}$ \\
ALT (n): & 75 & 5 & $0.002 \dagger$ \\
$\quad$ Yes & $4.7(3.2)$ & $8.3(3.3)$ & \\
$\quad$ No & $28.6(7.3)$ & $31.7(7.6)$ & $0.232 \dagger$ \\
Duration to operation (years) & $18.7(5.3)$ & $19.3(2.7)$ & $0.720 \dagger$ \\
After operation: & $1.2(0.9)$ & $1.5(0.9)$ & $0.340 \dagger$ \\
Untreated IOP & & & \\
Mean last IOP & & & \\
No of medicines & &
\end{tabular}

p Value $=$ significance level; IOP $=$ intraocular pressure; ALT $=$ argon laser trabeculoplasty; ^Fisher's exact test, $\uparrow t$ test.

Table 3 Characteristics at operation of patients included in the analysis of HRP visual field (SD)

\begin{tabular}{lccc}
\hline Characteristic & $\begin{array}{l}\text { Exfoliation glaucoma } \\
(n=16)\end{array}$ & $\begin{array}{l}\text { Simplex glaucoma } \\
(n=12)\end{array}$ & $p$ Value \\
\hline Age & $71.5(7.7)$ & $68.9(7.7)$ & 0.389 \\
IOP & $31.3(5.5)$ & $25.0(2.5)$ & 0.001 \\
No of medicines & $2.7(0.7)$ & $2.2(1.1)$ & 0.198 \\
Duration to operation & $4.9(4.0)$ & $8.2(5.4)$ & 0.078 \\
LogMAR & $0.2(0.3)$ & $0.2(0.3)$ & 0.548 \\
GD (dB) & $3.6(1.7)$ & $2.9(1.9)$ & 0.277 \\
NC (\%) & $29.5(19.9)$ & $35.5(30.0)$ & 0.530
\end{tabular}

$\mathrm{p}$ Value $=$ significance level; $\mathrm{IOP}=$ intraocular pressure MAR $=$ minimum angle of resolution; $\mathrm{GD}=$ global deviation; $\mathrm{NC}=$ neural channels.

Table 4 Characteristics at last visit of patients included in the analysis of HRP visual field (SD)

\begin{tabular}{lccc}
\hline Characteristic & $\begin{array}{l}\text { Exfoliation glaucoma } \\
(n=16)\end{array}$ & $\begin{array}{l}\text { Simplex glaucoma } \\
(n=12)\end{array}$ & p Value \\
\hline Follow up (months) & $46.2(16.4)$ & $45.0(17.1)$ & 0.854 \\
IOP & $15.3(5.1)$ & $17.7(3.2)$ & 0.166 \\
IOP reduction (\%) & $-50.0(17.0)$ & $-28.7(13.6)$ & 0.001 \\
Change in GD $(\mathrm{dB})$ & $+0.4(1.2)$ & $+0.3(1.5)$ & 0.854 \\
Change in NC† (\%) & $+1.6(13.1)$ & $+3.9(12.8)$ & 0.638 \\
Change in logMAR & $+0.1(0.3)$ & $0.0(0.2)$ & 0.181 \\
\hline
\end{tabular}

${ }^{\star}$ Mean postoperative GD - GD at operation; tmean postoperative NC - NC at operation; $\ddagger \log M A R$ at last visit $-\log M A R$ at operation. coma was 31.7 (22.8) months, and in simplex glaucoma $32.7(24.8)$ months $(\mathrm{p}=0.835)$.

A reinstatement of medical treatment was done in $42 \%$ of patients with exfoliation and in $36 \%$ of patients with simplex glaucoma $(p=0.673)$. Two patients with exfoliation and three patients with simplex glaucoma had, in addition, to be operated with new trabeculectomy. In all but three patients medical treatment was introduced owing to an unsatisfactory postoperative IOP level. Preoperative and postoperative characteristics in patients with added medication after operation are shown in Table 2. The IOP at operation, the untreated IOP, and the treated IOP at the end of follow up were similar for both glaucoma types. Among patients without postoperative medication the mean IOP at operation was 28.2 (5.4) $\mathrm{mm} \mathrm{Hg}$ in exfoliation and 24.8 (3.4) in simplex glaucoma $(\mathrm{p}=0.008)$, and at the end of follow up 14.3 (2.8) $\mathrm{mm} \mathrm{Hg}$ and 17.0 (3.7) $\mathrm{mm} \mathrm{Hg}(\mathrm{p}=0.002)$, respectively.

A correlation analysis between the last noted untreated IOP and the different preoperative characteristics (patients' age and IOP at operation, glaucoma type, sex, ALT, number of antiglaucoma eye drops and eye medicines, and length of time from glaucoma diagnosis to operation) was done by multivariate analysis. No significant correlation was found between the untreated IOP and any preoperative variable in patients with added medication after operation. In patients without any postoperative medication a significantly lower untreated IOP was found in exfoliation glaucoma $(r=400, \mathrm{p}=0.002)$.

The multivariate method was also used for analysis of correlation between the last untreated IOP regulation and the mode of conjunctival and scleral flap sutures. The two variables did not correlate significantly with untreated IOP regulation in any glaucoma type.

Mean age and mean IOP at operation as well as the distribution of glaucoma types were not significantly different between the patients included and patients excluded from visual field analysis. Fifty six per cent of the patients investigated with Goldmann perimetry had at operation extensive field defects in the form of absolute central, paracentral, or peripheral scotoma for $\mathrm{V}-4$ object involving central areas to 5 degree from the fixation point. Of these patients about $70 \%$ were patients with exfoliation glaucoma. Two patients with simplex glaucoma suffered from marked progress of field defects despite a satisfactory IOP decrease. In one of these patients magnetic resonance tomography showed a cerebral atrophy.

Preoperative characteristics of patients included in the HRP visual field analysis are shown in Table 3, and their characteristics at last visit are given in Table 4 .

Mean change in GD per year of postoperative follow up was $0.16(0.4) \mathrm{dB}$ for exfoliation and $0.08(0.4) \mathrm{dB}$ for simplex glaucoma $(\mathrm{p}=$ 0.560 ). At the last visit GD had deteriorated in $63 \%$ of patients with exfoliation and in $67 \%$ of patients with simplex glaucoma. Figure 2 shows a scatter plot of GD $v$ postoperative follow up time in patients with deteriorating fields of both 


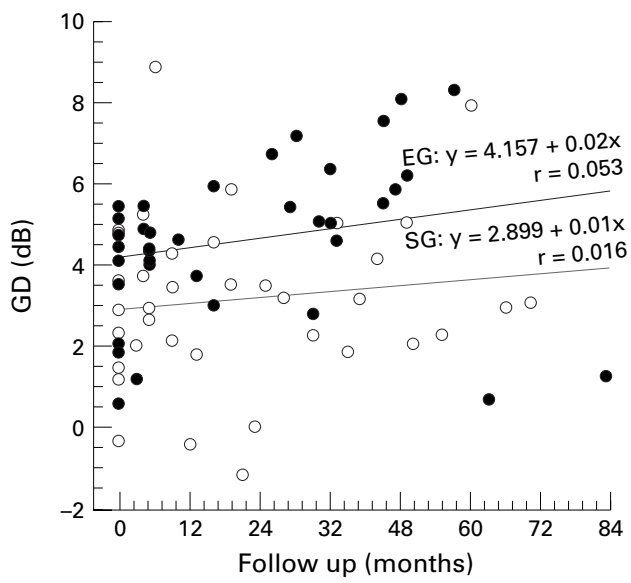

Figure 2 Scatter plot of global deviation (GD) in patients with deteriorated visual field according to glaucoma type. $\bullet$ = exfoliation glaucoma (EG), $\mathrm{O}=$ simplex glaucoma $(S G)$.

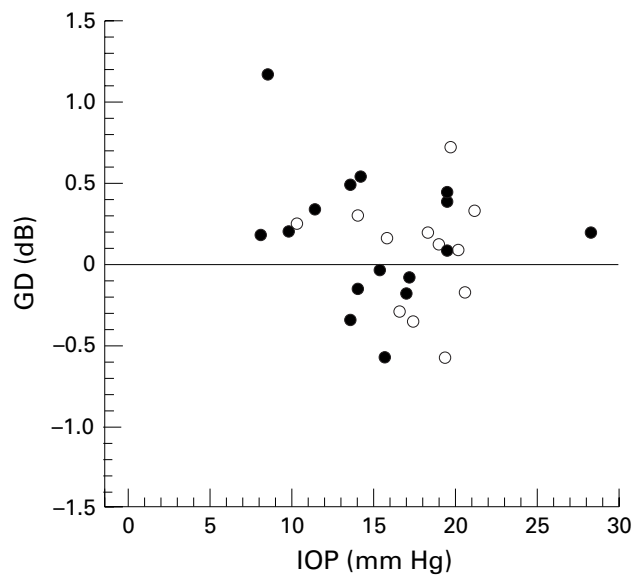

Figure 3 Plot of mean change in global deviation (GD) per year of postoperative follow up $v$ IOP at last visit according to glaucoma type. $\bullet=$ exfoliation glaucoma $\mathrm{O}=$ simplex glaucoma .

glaucoma types. Starting from different levels at base line the least square fit for the two groups showed a decay with time of a similar magnitude. The linear trend was not significant for any glaucoma type (slope 0.02, p $=0.166$ for exfoliation and slope $0.013, p=0.457$ for simplex glaucoma), nor was there significant difference between the trend of the two lines $(\mathrm{p}=0.729)$.

Mean change in postoperative GD per year of follow up $v$ IOP at last visit for all 28 patients is given in Figure 3. Mean IOP at last visit in patients with improved GD was 16.6 (2.1) $\mathrm{mm}$ $\mathrm{Hg}$ and in patients with deteriorated GD 16.2 (5.4) $\mathrm{mm} \mathrm{Hg}(\mathrm{p}=0.801)$. Mean percentage of IOP reduction in patients with unchanged/ improved and deteriorated GD after operation was $39.5 \%(13.2 \%)$ and $41.7 \%(21.4 \%)(\mathrm{p}=$ $0.775)$, respectively.

Among those with deterioration of GD $(n=18)$ the mean change of NC per year was $-1.5 \%(3.0 \%)$ in exfoliation and $-0.3 \%$ $(2.5 \%)$ in simplex glaucoma $(p=0.392)$. Two cases of both glaucoma types showed an anomalous pattern with an improvement of NC $(2-3 \%)$ in spite of deterioration of GD of in average $0.25 \mathrm{~dB}$.
The pattern of complications was similar in both glaucoma types. The most common complication was cataract progression $(60 \%$ in exfoliation and $48 \%$ in simplex glaucoma, $\chi^{2}=$ $3.7, p=0.298)$. Thirty two per cent of patients with exfoliation glaucoma and $19 \%$ of patients with simplex glaucoma were operated for cataract following trabeculectomy. Fisher's exact test reveals no significant dependence between cataract surgery and glaucoma types $(\mathrm{p}=0.164)$. In relation to sex, cataract surgery was performed in $29 \%$ of males and in $24 \%$ of females $(p=0.820)$.

\section{Discussion}

The rates of exfoliation (56\%) and simplex glaucoma $(44 \%)$ in the present study are similar to the rates of these glaucoma types in a previous study from Scandinavia. ${ }^{11}$ The higher rates of exfoliation glaucoma in these two Scandinavian studies in comparison with the study of Konstas et al ${ }^{12}$ may indicate regional differences in prevalence of the two glaucoma types.

In comparison with simplex glaucoma (Table 1), patients with exfoliation glaucoma were older and suffered from more aggressive glaucoma before the operation. The aggressiveness of exfoliation glaucoma is reflected by a higher IOP, more topical antiglaucoma drugs, and a shorter duration to operation. The shorter duration to operation in exfoliation glaucoma was again demonstrated in patients with added medication after operation (Table 2) and the higher preoperative IOP in the subsample of patients who underwent visual field analysis (Table 3).

Concerning IOP regulation after operation, two groups of patients were identified. The first group consists of patients with need for addition of medication after operation to achieve satisfactory IOP regulation. In these patients the mean IOP at operation, the last noted postoperative untreated IOP, and the mean IOP at the end of follow up were similar for both glaucoma types. The second group consists of patients in whom the IOP was controlled during the whole postoperative follow up without any added treatment. Among these patients the mean IOP in exfoliation glaucoma was significantly higher at operation and significantly lower at the end of follow up.

In previously published studies on different glaucoma types, the rate of long term IOP control after primary trabeculectomy without antimetabolites varies from $40 \%$ to $95 \% .^{12} 1415$ Among other factors, the efficacy of trabeculectomy on IOP regulation in chronic open angle glaucoma may be influenced by patient age, glaucoma types, number of antiglaucoma medications, and duration to operation. ${ }^{16-18}$

There are very few studies in which the effect of trabeculectomy in exfoliation and simplex glaucoma is compared. In the study by Konstas et $a l^{12}$ the untreated IOP was significantly lower in exfoliation than in simplex glaucoma 6 months after operation. Our findings on medicine free time after operation in all patients in the study and untreated IOP regulation among patients with added postoperative medication, 
after on average 46.2 months of follow up, indicate that the effect of trabeculectomy alone is similar for both glaucoma types (Table 2, Fig 1). However, the IOP regulation in patients without any medication postoperatively was in agreement with the results in the above cited study. ${ }^{12}$

Visual field examination has an essential role in diagnosis and follow up of glaucoma. Results are influenced by many factors-for example, patient age, media opacities, visual acuity, perimetry technique, patient reliability, and selection of subsample. In the subsample of patients who underwent visual field analysis in the present study, perimetry was performed by $\mathrm{HRP}$, a technique found to be comparable with Humphrey perimetry in detection and follow up of glaucomatous visual field defects. ${ }^{19}{ }^{20} \mathrm{To}$ minimise the influence of cataract on visual field results we excluded from the analysis all visual fields of patients with cataract denser than early stage opacities. Other factors that may affect the calculation of the deterioration during follow up may be the effect of training or the effect of aging as described by Martin and Wanger $^{21}$ using the same perimetric methodology. The effect of age, however, is adjusted in the calculation of GD. ${ }^{22}$

About two thirds of the cases showed visual field deterioration in both types of glaucoma. The magnitude of GD deterioration per year was higher (eight times in exfoliation and four times in simplex glaucoma) than the expected age related deterioration. In a 5 year follow up of cases on medical antiglaucoma treatment, Martin and Wanger ${ }^{21}$ found that $45 \%$ deteriorated and that the visual field deterioration was on average $0.41 \mathrm{~dB}$ per year-that is, more than 20 times the expected age related deterioration.

The lower rates of field deterioration in our study cannot be explained by more efficacious IOP lowering in comparison with the above cited study. The findings that the average IOP was at the same level (about $16 \mathrm{~mm} \mathrm{Hg}$ ) in the group with and without visual field deterioration, and that GD change per year was unrelated to IOP level or IOP reduction, indicate that factors other than IOP affect visual field decay.

Martin and Wanger ${ }^{21}$ found that the visual field deterioration was preceded by a transient improvement during the first 2 years after the initiation of antiglaucoma treatment. This effect was rare in our study and was noted only at the first postoperative investigation in a few patients with continued field loss. This may be explained by the fact that none of our patients had newly diagnosed glaucoma, and all were used to visual field investigation from the time before trabeculectomy.

In HRP NC generally increases when the GD level decreases and vice versa. GD is an index that reflects the state of the whole field inside 30 degrees, both within its central and peripheral part. NC accounts for changes mostly in the central part of the visual field owing to the fact that most of the neural channels are located centrally. ${ }^{13}$ The difference between the two indices may explain the finding of obvious deterioration of GD associated with improvement of NC in some patients. In these patients the most central part of the visual field remained stable while the peripheral part deteriorated.

In our study the treated IOP at last visit was better controlled in exfoliation than in simplex glaucoma. However, the untreated IOP and visual field survival were similar for both types of glaucoma. Therefore, the present study does not support the findings in the study of Törnqvist and Drolsum, ${ }^{11}$ in which the trend for progression of visual field decay and optic disc changes after operation was significantly lower in patients with exfoliation than in patients with simplex glaucoma. However, the results in these two studies may not be fully comparable owing to difference in outcome measures.

Cataract progression was found to be the most common late complication after trabeculectomy, in agreement with previous reports. ${ }^{23}{ }^{24}$ In the study of Vesti ${ }^{25}$ cataract was more accelerated in exfoliation than in primary open angle glaucoma. The criteria for acceleration of cataract in the above cited study ${ }^{25}$ were the number of cases with cataract extraction, the shift of refraction to myopia, and increase in the lens opacity value measured with a Lens Opacity Meter 701. In the present study cataract progression was assessed by slit lamp investigation and ophthalmoscopy with a dilated pupil and the rates for progression were not significantly different between the two glaucoma types.

In summary, patients with exfoliation glaucoma were older and suffered from more aggressive glaucoma before operation compared to patients with simplex glaucoma. The IOP lowering effect of trabeculectomy alone was similar for both types of glaucoma. A good IOP regulation did not always imply a successful operation with respect to visual field survival. The visual field survival was similar for both glaucoma types.

1 Popovic V, Sjöstrand J. Long-term outcome following trabeculectomy. I Retrospective analysis of intraocular pressure egulation and cataract formation. Acta Ophthalmol (Copenh) 1991;69:299-304.

2 Watson PG, Jakeman M, Ozturk M, et al. The complications of trabeculectomy (a 20-year follow-up). Eye 1990;4:42538

3 Aasved $\mathrm{H}$. The frequency of fibrillopathia epitheliocapsularis (so-called senile exfoliation or pseudoexfoliation) in patients with open-angle glaucoma. Acta Ophthalmol (Copenh) 1971;49:1-17.

4 Olivius E, Thorburn W. Prognosis of glaucoma simplex and glaucoma capsulare. A comparative study. Acta Ophthalmol (Copenh) 1978;56:921-34.

5 Pohjanpelto P. Long-term prognosis of visual field in glaucoma simplex and glaucoma capsulare. Acta Ophthalmol (Copenh) 1985;63:418-23.

6 Airaksinen PJ. The long-term hypotensive effect of timolol maleate compared with the effect of pilocarpine in simple and capsular glaucoma. Acta Ophthalmol (Copenh) 1979; 57:425-34

7 Bilka S, Saunte E. Timolol maleate in the treatment of glaucoma simplex and glaucoma capsulare. Acta Ophthalmol (Copenh) 1982;60:967-76.

8 Bergeå B. Intraocular pressure reduction after argon laser trabeculoplasty in open-angle glaucoma. A two-year follow-up. Acta Ophthalmol (Copenh) 1986;64:401-6.

9 Threlkeld AB, Hertzmark E, Sturm RT, et al. Comparative study of the efficacy of argon laser trabeculoplasty for exfoliation and primary open-angle glaucoma. 7 Glaucoma 1966;5:311-16.

10 Jerndal T, Kriisa V. Results of trabeculectomy for pseudoexfoliative glaucoma. A study of 52 cases. Br f Ophthalmol 1974;58:927-30. A study of 52 cases. Br f Ophthalmol

11 Törnqvist G, Drolsum LK. Trabeculectomies. A long-term study. Acta Ophthalmol Copenh) 1991;69:450-4. 
12 Konstas AGP, Jay JL, Marshall GE, et al. Prevalence, diagnostic features, and response to trabeculectomy in

13 Frisén L. Acuity perimetry: estimation of neural channels. Int Ophthalmol 1988;12:169-174.

14 Nouri-Mahdavi K, Brigatti L, Weitzman M, et al. Outcomes of trabeculectomy for primary open-angle glaucoma. $O p h-$ thalmology 1995;102:1760-9.

15 Chen TC, Wilensky JT, Viana MAG. Long-term follow-up of initially successful trabeculectomy. Ophthalmology 1997 104:1120-5.

16 Gressel MG, Heuer DK, Parrish RK. Trabeculectomy in young patients. Ophthalmology 1984;91:1242-6.

17 Lavin MJ, Wormald RPL, Migdal CS, et al. The influence of prior therapy on the success of trabeculectomy. Arch Ophthalmol 1990;108:1543-8.

18 Jay JL, Murray SB. Early trabeculectomy versus conventional management in primary open angle glaucoma. $\mathrm{Brf}$ tional management in primart
19 Martinez GA, Sample PA, Weinreb RN. Comparison of high-pass resolution perimetry and standard automated perimetry in glaucoma. Am F Ophthalmol 1995;119:195201.

20 Martin-Boglind L, Wanger P. The effect of treatment on the results of high-pass resolution perimetry in glaucoma. Acta Ophthalmol (Copenh) 1994;72:423-8.

21 Martin L, Wanger P. Five-year follow-up of treated patients with glaucoma using resolution perimetry. $\mathcal{F}$ Glaucoma with glaucom

22 Ophthimus System Manual. 2nd ed. Malmö, Sweden: High Tech Vision, 1990

23 Popovic V. Early hypotony after trabeculectomy. Acta Ophthalmol (Copenh) 1995;73:255-60.

24 Popovic V. Early choroidal detachment after trabeculectomy. Acta Ophthalmol (Copenh) 1998;76:367-71.

25 Vesti E. Development of cataract after trabeculectomy. Acta Ophthalmol (Copenh) 1993;71:777-81. 